\title{
Charge Carriers Are Not Affected by the Relatively Slow-Rotating Methylammonium Cations in Lead Halide Perovskite Thin Films
}

\author{
Valentina M. Casellii, ${ }^{\dagger}$ Mathias Fischer, ${ }^{\ddagger}$ Daniele Meggiolaro, ${ }^{\S}$ Edoardo Mosconi, ${ }^{\S}$ \\ Filippo De Angelis, ${ }^{\S}$ Samuel D. Stranks, ${ }^{\Perp}$ Andreas Baumann, ${ }^{\perp}{ }^{\perp}$ Vladimir Dyakonov, ${ }^{\ddagger}$ \\ Eline M. Hutter, $*, * \#$ and Tom J. Savenije ${ }^{*, \dagger}$ \\ ${ }^{\dagger}$ Department of Chemical Engineering, Delft University of Technology, $2629 \mathrm{HZ}$ Delft, The Netherlands \\ ${ }^{\ddagger}$ Experimental Physics 6, Julius-Maximillian University of Würzburg, Am Hubland, D-97074 Würzburg, Germany \\ ${ }^{\S}$ Computational Laboratory for Hybrid/Organic Photovoltaics, CNR-ISTM and Department of Chemistry, Biology and \\ Biotechnology, University of Perugia, Via Elce di Sotto 8, I-06123, Perugia, Italy \\ "Cavendish Laboratory, University of Cambridge, JJ Thomson Avenue, Cambridge CB3 0HE, United Kingdom \\ ${ }^{\perp}$ Bavarian Center for Applied Energy Research (ZAE Bayern), Magdalene-Schoch-Str. 3, D97074 Würzburg, Germany \\ ${ }^{\#}$ Center for Nanophotonics, AMOLF, Science Park 104, 1098 XG Amsterdam, The Netherlands
}

\section{Supporting Information}

ABSTRACT: Recently, several studies have investigated dielectric properties as a possible origin of the exceptional optoelectronic properties of metal halide perovskites (MHPs). In this study we investigated the temperature-dependent dielectric behavior of different MHP films at different frequencies. In the gigahertz regime, dielectric losses in methylammonium-based samples are dominated by the rotational dynamics of the organic cation. Upon increasing the temperature from 160 to $300 \mathrm{~K}$, the rotational relaxation time, $\tau$, decreases from 400 (200) to 6 (1) ps for $\mathrm{MAPb}_{3}\left(-\mathrm{Br}_{3}\right)$. By contrast, we found negligible temperature-dependent variations in $\tau$ for a mixed cation/mixed halide $\mathrm{FA}_{0.85} \mathrm{MA}_{0.15} \mathrm{~Pb}$ $\left(\mathrm{I}_{0.85} \mathrm{Br}_{0.15}\right)_{3}$. From temperature-dependent time-resolved microwave conductance

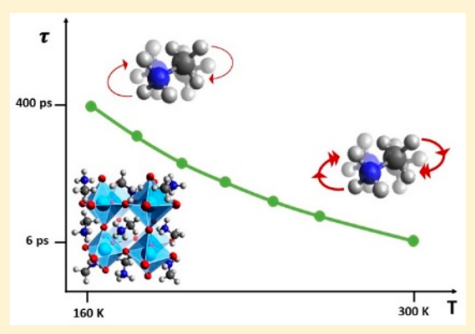
measurements we conclude that the dipolar reorientation of the MA cation does not affect charge carrier mobility and lifetime in MHPs. Therefore, charge carriers do not feel the relatively slow-moving MA cations, despite their great impact on the dielectric constants.

T $\mathrm{n}$ the past few years, solar cells based on metal halide 1 perovskite (MHP) semiconductors, with general structure $\mathrm{ABX}_{3}$, have emerged as promising low-cost alternatives to established semiconductors like silicon and CIGS. In addition, other applications such as light-emitting diodes, lasers and photodetectors were recently reported. ${ }^{1}$ The success of MHPbased solar cells is due to their exceptional optoelectronic properties, such as high absorption coefficients, low exciton binding energies, and long charge carrier lifetimes and diffusion lengths. ${ }^{2-5}$ Part of these properties can be attributed to the low densities of trap states and background charges. ${ }^{5,6}$ In addition, it has been suggested that the large dipole moment of the methylammonium group $\left(\mathrm{MA}^{+}\right)$in $\mathrm{MAPbI}_{3}$ can result in the formation of ferroelectric domains by the collective motion of the $\mathrm{MA}^{+}$dipoles. ${ }^{7,8}$ This, although still under debate, might lead to enhanced exciton discociation, polaron formation, ${ }^{9}$ reduced charge carrier recombination, and defect screening. $^{10-12}$ The rotational dynamics of the MA cation have been theoretically and experimentally studied. ${ }^{11,13-21}$ First studies on the rotational dynamics were carried out using $\operatorname{IR}^{19}$ and dielectric $^{22}$ techniques. IR measurements on powders revealed a very rapid reorientation of the MA cation around the $\mathrm{C}-\mathrm{N}$ axis with activation energies of 2.6 and $2.0 \mathrm{~kJ} / \mathrm{mol}$ and relaxation times 1 and 0.7 ps for $\mathrm{MAPbI}_{3}$ for $\mathrm{MAPbBr}_{3}$, respectively. ${ }^{19}$ On the other hand, from dielectric measurements at $90 \mathrm{GHz}$, Poglitsch and Weber determined relaxation times of 5.37 and 2.73 ps for $\mathrm{MAPbI}_{3}$ and $\mathrm{MAPbBr}_{3}$, respectively. $^{22}$ As extensively discussed by Gallop et al., ${ }^{12}$ these values can be attributed to two different motions, namely, a rapid wobbling and a slower reorientation of the $\mathrm{MA}^{+}$group. Raman spectroscopy has been more recently applied to study the dynamic (dis)order in perovskite materials. While the link between the (dis)order of the MA cation and the orthorhombic-tetragonal phase transition in $\mathrm{MA}^{+}$-based MHPs appears to be clear, ${ }^{11,20}$ its influence on the lattice dynamics is still under debate. ${ }^{12,23}$ Furthermore, the impact of $\mathrm{MA}^{+}$dipole on the formation of ferroelectric domains is still under discussion, although recent dielectric ${ }^{11}$ and impedance spectroscopy (IS) ${ }^{21}$ studies reported no indication of formation of ferroelectric domains in these compounds. Theoretical modeling confirmed the limited stability of a possible ferroelectric $\mathrm{MAPbI}_{3}$ phase at room temperature. $^{24,25}$

Received: July 24, 2019

Accepted: August 9, 2019

Published: August 9, 2019 

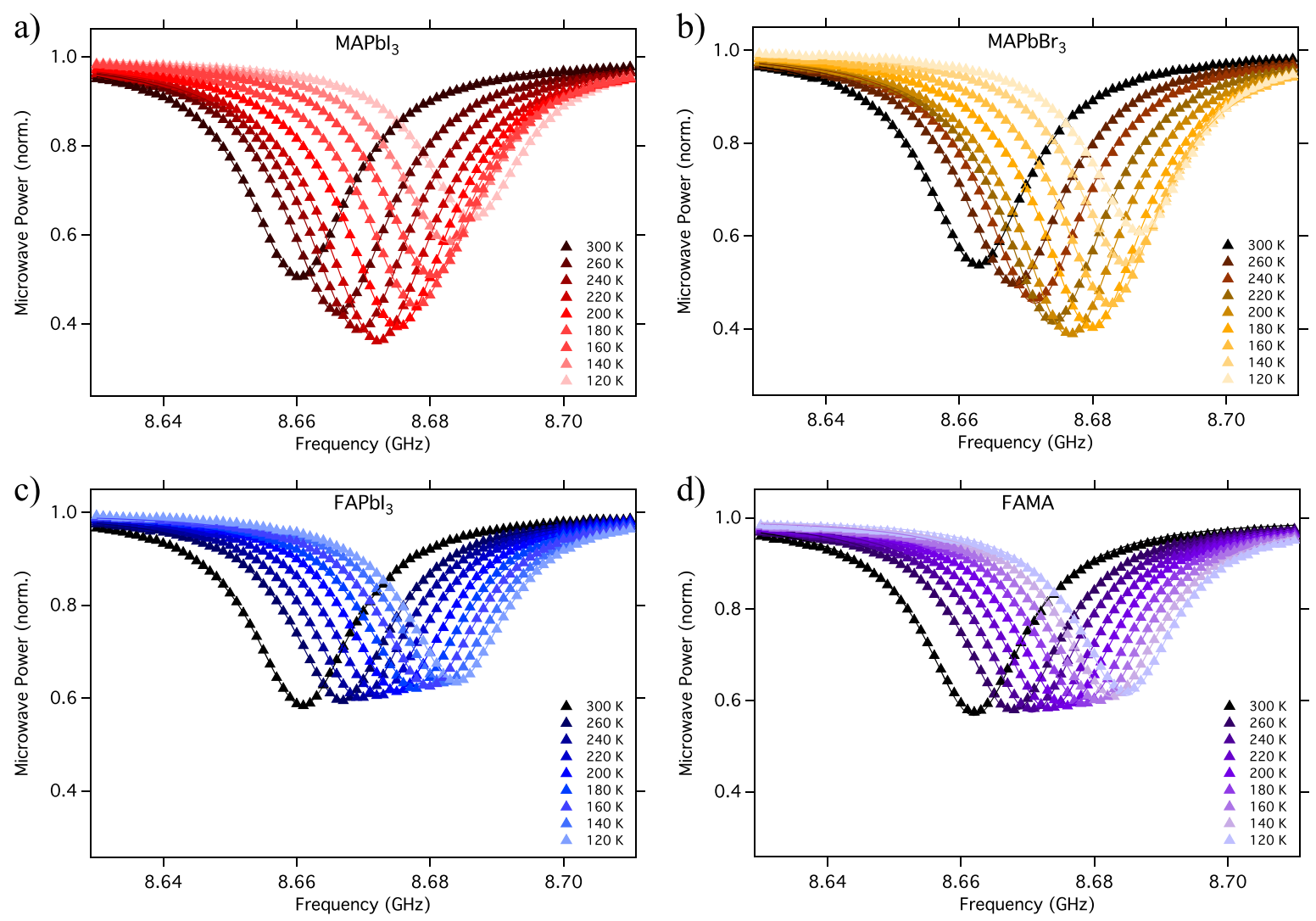

Figure 1. Normalized microwave power as a function of frequency recorded at different temperatures for (a) $\mathrm{MAPbI}_{3},(\mathrm{~b}) \mathrm{MAPbBr}_{3},(\mathrm{c}) \mathrm{FAPbI}_{3}$, and (d) $\mathrm{FA}_{0.85} \mathrm{MA}_{0.15} \mathrm{~Pb}\left(\mathrm{I}_{0.85} \mathrm{Br}_{0.15}\right)_{3}$ (FAMA). The solid lines are the results of the fits to the experimental data points (triangles).

Despite the above-mentioned studies on dipolar motion in MHPs, knowledge of dielectric properties in the gigahertz $(\mathrm{GHz})$ regime is still limited. Poglitsch and Weber ${ }^{22}$ and Anusca et al. ${ }^{11}$ have shown the great impact of temperature on dielectric properties of these materials, but both studies are missing information around $10 \mathrm{GHz}$. In this range, substantial changes in the temperature-dependent dielectric behavior related to the presence of the $\mathrm{MA}^{+}$dipolar reorientational motion can be expected. Moreover, to the best of our knowledge, no such study has been devoted to MHPs with different cations.

In this study we investigate the relationship between the perovskite constituents, temperature, and the dielectric properties by means of microwave conductance (MC) measurements in the $\mathrm{GHz}$ regime. All these measurements are performed in the dark in contrast to standard time-resolved microwave conductance (TRMC) measurements in which light-induced changes in the real and imaginairy parts of the dielectric constant of many materials have been studied, revealing the time-dependent formation of, for example, charge-transfer states, $^{26}$ excitons, ${ }^{27}$ and mobile charges. ${ }^{6}$ The high quality factor of our cavity with resonance frequencies around $10 \mathrm{GHz}$ ensures that even small changes in the dielectric properties by, for example, changing the constituents of the MHPs can be determined. Next, we prepared devices from the corresponding layers by using indium tin oxide (ITO) and $\mathrm{Au}$ as contact layers and recorded the real and imaginairy dielectric properties by IS betweeen $20 \mathrm{~Hz}$ and $2 \mathrm{MHz}$ in the dark.

We performed MC measurements on the following spincoated MHP layers: $\mathrm{MAPbI}_{3}, \mathrm{MAPbBr}_{3}, \mathrm{FAPbI}_{3}, \mathrm{CsPbI}$, and $\mathrm{FA}_{0.85} \mathrm{MA}_{0.15} \mathrm{~Pb}\left(\mathrm{I}_{0.85} \mathrm{Br}_{0.15}\right)_{3}$ (FAMA). Interestingly, MA-based
MHPs exhibited a strong temperature-dependent dielectric loss in the $\mathrm{GHz}$ regime, which is absent for the FA- and Csbased MHPs. With the low-frequency IS measurements, no appreciable temperature-dependent changes were observed for both $\mathrm{MAPbI}_{3}$ and $\mathrm{FAPbI}_{3}$, meaning that the dielectric loss in the $\mathrm{GHz}$ regime is not related to background charges. Therefore, we attribute the changes in dielectric losses to the rotational motion of the $\mathrm{MA}^{+}$dipoles. Activation energies for the rotational motion and corresponding temperature-dependent relaxation times for $\mathrm{MAPbI}_{3}, \mathrm{MAPbBr}_{3}$, and FAMA were obtained by applying the Cole-Cole model $^{28}$ to our results. Despite the great impact of the dipolar orientation on dielectric properties in the $\mathrm{GHz}$ regime, no major implications for the charge carrier dynamics were found.

MHP samples were fabricated on quartz or on patterned ITO by the two step spin-coating procedure followed by a 5 min annealing step at $100{ }^{\circ} \mathrm{C}$. For optical and structural characterization see Figure S1 of the Supporting Information. For the MC technique an MHP layer on quartz was mounted in a temperature-controlled microwave cavity without any exposure to air. Apart from its superior sensitivity, the MC technique does not require electrical contacts. First, a scan of the reflected microwave power is recorded by sweeping the frequency, $v$, over a certain microwave range reveiling a dip, which can be attributed to the formation of a standing wave pattern within the loaded cavity. Next, the microwave scans are normalized to a scan recorded by replacing the cavity with a fully reflecting end plate. In Figure 1 the normalized scans of $\mathrm{MAPbI}_{3}, \mathrm{MAPbBr}_{3}, \mathrm{FAPbI}_{3}$, and FAMA are shown in a temperature range varying from 120 to $300 \mathrm{~K}$. The gradual shift to lower frequencies upon heating combined with the 

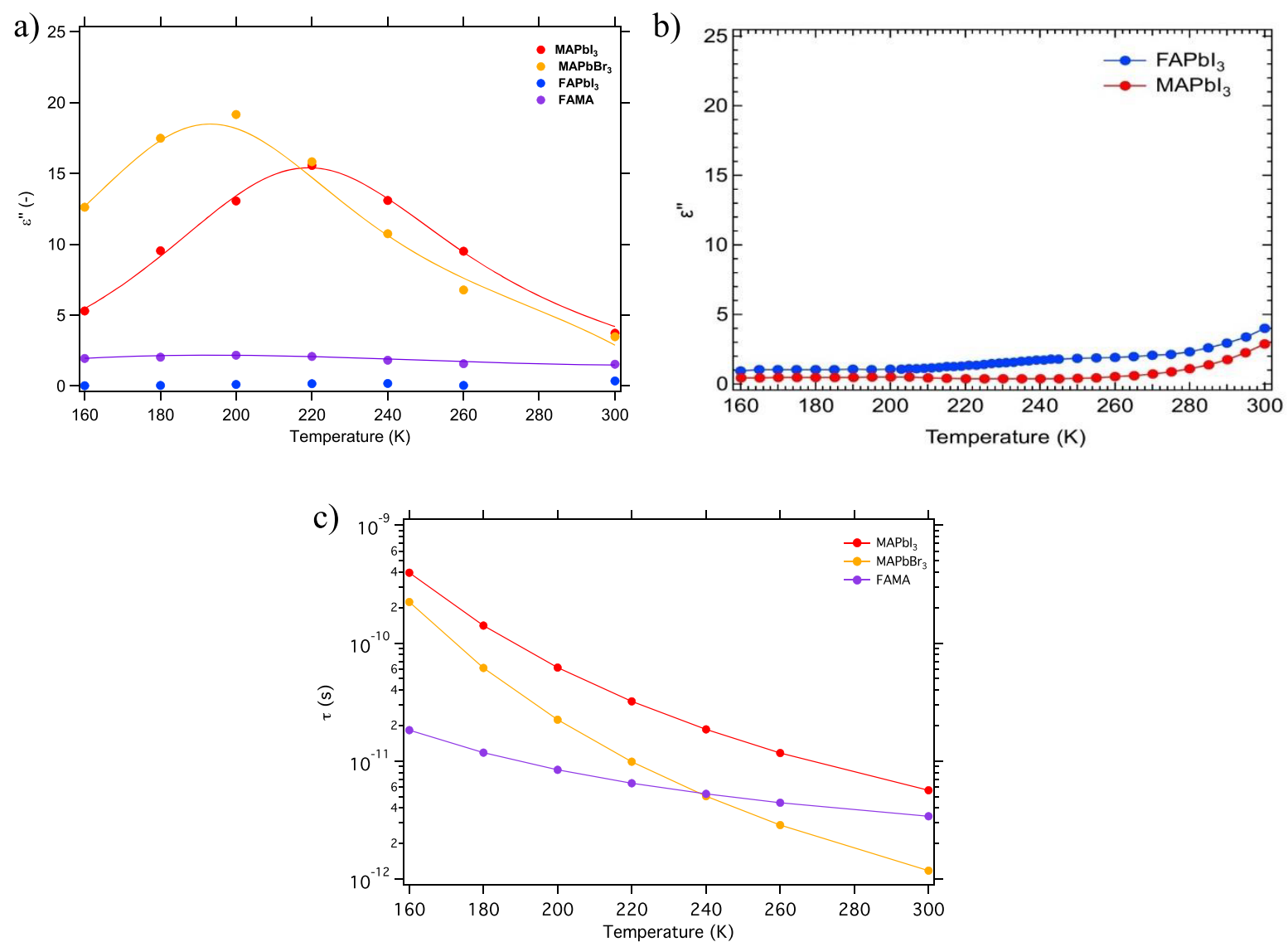

Figure 2. Imaginary part of the complex dielectric constant (a) from MC measurements at $8.6 \mathrm{GHz}$ and (b) at $1 \mathrm{kHz}$ obtained by impedance spectroscopy. (c) Relaxation times for the $\mathrm{MA}^{+}$containing MHPs.

increase in microwave absorption can be explained by changes of the cavity, i.e. expanding of the cavity length and enhanced losses in the metallic walls, respectively (see the Supporting Information for more details). Most importantly, the scans of the MA-based samples are substantially different from those of $\mathrm{FAPbI}_{3}$ and $\mathrm{CsPbI}_{3}$. That is, up to $220 \mathrm{~K}$ for $\mathrm{MAPbI}_{3}$ and up $200 \mathrm{~K}$ for $\mathrm{MAPbBr}_{3}$, we observe an increase of microwave absorption, which then decreases again at higher temperatures. For $\mathrm{FAPbI}_{3}$ and $\mathrm{CsPbI}_{3}$, on the other hand, the scans are very similar to those observed for a bare quartz substrate (see Figure S2b).

The power scans in Figure 1 were modeled using a custombuilt computer program, which numerically solves the Maxwell equations using the resonance characteristics of the cavity and the dielectric properties and dimensions of all media inside the cell as input. ${ }^{29}$ The results of the fits are shown as solid lines in Figure 1, and the temperature-dependent conductivities are summarized in Table S1 in the Supporting Information. The values of the imaginary dielectric constant, $\varepsilon^{\prime \prime}$, are calculated from the real conductivities, $\sigma_{\mathrm{Re}}$, by

$$
\sigma_{\mathrm{Re}}=\omega \varepsilon_{0} \varepsilon^{\prime \prime}
$$

where $\omega$ is the angular frequency and $\varepsilon_{0}$ the vacuum permittivity (Figure 2a). For both MA-based MHPs a clear maximum is observed, while $\mathrm{FAPbI}_{3}$ and FAMA show almost no temperature dependence.

We note that absorption of microwaves by MHPs can have multiple origins, including the presence of mobile charge carriers, of mobile ions, and/or dipolar reorientation-related losses. For this reason, we conducted additional IS measure- ments on $\mathrm{MAPbI}_{3}$ and $\mathrm{FAPbI}_{3}$ over a wide frequency range, from $20 \mathrm{~Hz}$ to $2 \mathrm{MHz}$. At frequencies of $10 \mathrm{kHz}$ and above, $\varepsilon^{\prime \prime}$ is already influenced by series resistance resulting from the electrical contacts ITO and Au (see Figure S7). Therefore, the resulting relative value of $\varepsilon^{\prime \prime}$ as a function of temperature at 1 $\mathrm{kHz}$ is shown for $\mathrm{FAPbI}_{3}$ and $\mathrm{MAPbI}_{3}$ in Figure $2 \mathrm{~b}$. In contrast to the $\mathrm{MC}$ results, the temperature dependence of $\varepsilon^{\prime \prime}$ determined by IS for $\mathrm{MAPbI}_{3}$ and $\mathrm{FAPbI}_{3}$ are similar and the specific maximum observed at $220 \mathrm{~K}$ in the $\mathrm{GHz}$ regime for $\mathrm{MAPbI}_{3}$ (Figure 2a) is not present at kilohertz $(\mathrm{kHz})$ frequencies (Figure $2 \mathrm{~b}$ ). In addition, we observe an increase of $\varepsilon^{\prime \prime}$ with temperature for both devices in the $\mathrm{kHz}$ regime, which we ascribe to temperature-activated ionic conductivity $^{30,31}$ and which is not observed in MC. These dissimilarities between the MC and IS measurements provide evidence against the hypothesis that temperature-dependent dielectric losses in the $\mathrm{GHz}$ range are due to mobile species such as charges or mobile ions. The observation that only the MAbased MHPs show a dielectric behavior that is considerably different than that of a quartz reference substrate implies that the dipolar reorientation of the MA dipolar cation is responsible for the distinctive temperature dependence of $\varepsilon^{\prime \prime}$ shown in Figure 2a.

Previously the dielectric behavior of the $\mathrm{MA}^{+}$-based MHPs has been modeled successfully to extract relaxation times and activation energies of MA reorientation using the Cole-Cole model, ${ }^{28}$ in which the complex dielectric constant $\varepsilon^{*}$ is defined as 

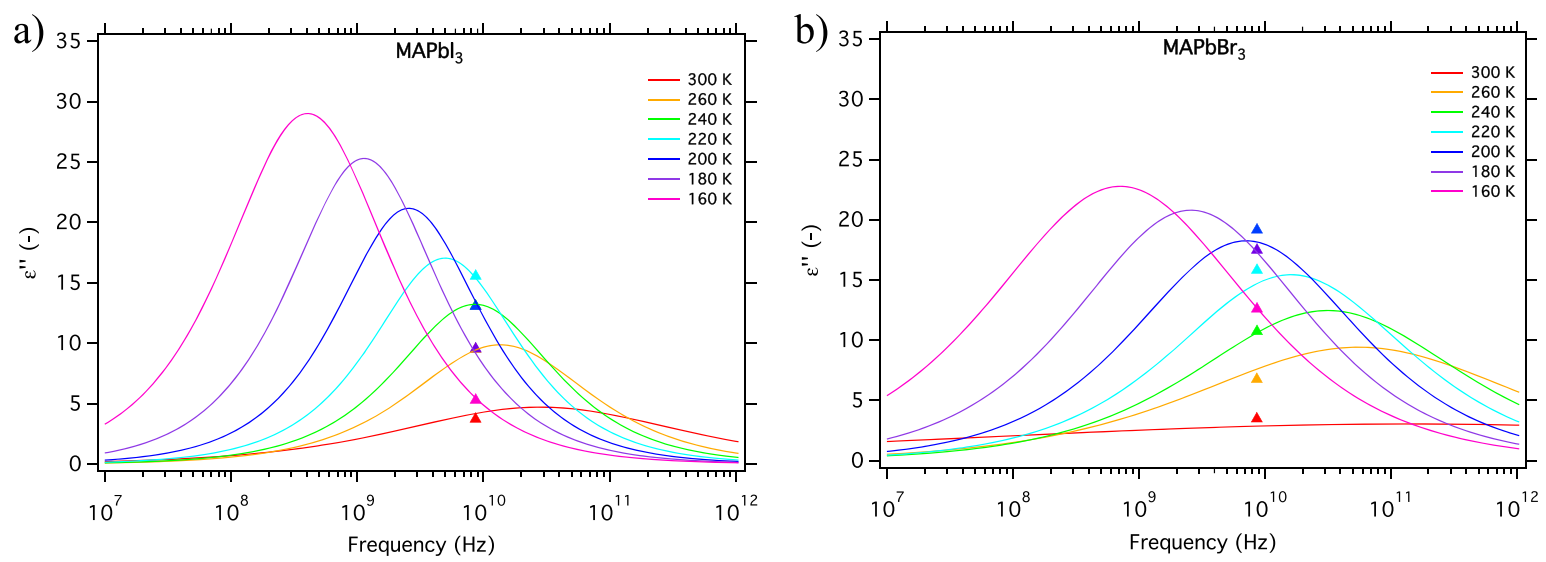

Figure 3. Calculated values of the dielectric losses at different temperatures versus frequency for (a) $\mathrm{MAPbI}_{3}$ and (b) $\mathrm{MAPbBr}_{3}$ by applying the Cole-Cole equation. The triangles indicate the experimental data points.

$$
\varepsilon^{*}=\varepsilon_{\infty}+\frac{\varepsilon_{\mathrm{S}}-\varepsilon_{\infty}}{1+(\mathrm{i} \omega \tau)^{(1-\alpha)}}
$$

where $\varepsilon_{\infty}$ and $\varepsilon_{\mathrm{S}}$ represent the high-frequency and static dielectric constants, respectively; $\tau$ represents the specific relaxation time, and $\alpha$ is a parameter reflecting the width of the relaxation time distribution. The specific expressions for the real and imaginary parts are given in the Supporting Information. The temperature dependence of the static and high-frequency dielectric constants are derived from the IS (at $1 \mathrm{kHz}$ ) and $\mathrm{MC}$ (fitting in the range 8.6-8.7 GHz) measurements, respectively. Values are collected in Table S1 in the Supporting Information. The relaxation time, $\tau$, is defined as

$$
\tau=\tau_{0} \exp \left(\frac{E_{\mathrm{A}}}{k_{\mathrm{B}}\left(T-T_{0}\right)}\right)
$$

in which $\tau_{0}$ is the relaxation time at high temperatures, $E_{\mathrm{A}}$ the activation energy, and $T_{0}$ the temperature at which the relaxation time could be considered as "infinitely slow". 32,33

The experimental $\varepsilon^{\prime \prime}$ data points are fitted with the ColeCole equation, and the resulting fits are added in Figure 2a, showing excellent overlap for both $\mathrm{MAPbI}_{3}$ and $\mathrm{MAPbBr}_{3}$.

We notice that for $\mathrm{MAPbBr}_{3}$ the model is able to fit the results even in the cubic phase $(T>235 \mathrm{~K})$ with minimal discrepancies. When a prefactor of 0.41 for the FAMA sample is included, a proper match between experimental data points and fit is observed. This experimentally obtained number accounts for different factors, i.e., the reduced $\mathrm{MA}^{+}$ concentration in the sample, $\mathrm{MA}^{+}-\mathrm{FA}^{+}$interactions, and the influence of the mixed halide. Proper quantification of each of these factors in such a complex system would require analyses and computational studies that go beyond the scope of this work. Despite the fact that $\tau$ of the FA cations should be in the same range as those of MA cations, ${ }^{12}$ our analysis could not be applied to the $\mathrm{FAPbI}_{3}$ results because of the low magnitude of the dielectric losses. The difference with $\mathrm{MA}^{+}$-based MHPs can be explained on the basis of the reduced dipolar character of FA compared to MA cations, which implies a significantly reduced response to the applied electric field in the former system.

The temperature-dependent relaxation times for the $\mathrm{MA}^{+}$based MHPs are shown in Figure 2c. Values at room temperature vary from 1 ps for $\mathrm{MAPbBr}_{3}$ to $6 \mathrm{ps}$ for
$\mathrm{MAPbI}_{3}$ and are comparable to those previously determined on compressed powders by neutron scattering ${ }^{17}$ and dielectric measurements. ${ }^{1,22}$ Activation energies of 114,135 , and 37 $\mathrm{meV}$ were determined for the rotational motion of $\mathrm{MAPbI}_{3}$, $\mathrm{MAPbBr}_{3}$, and FAMA, respectively. The increase in $E_{\mathrm{A}}$ from $\mathrm{MAPbI}_{3}$ to $\mathrm{MAPbBr}_{3}$ can be related to the increased hydrogen bonding strength and reduction of the octahedral cavity size, ${ }^{12}$ as confirmed by XRD measurements (see Figure S1b). DFT calculations (see computational details section in the Supporting Information) also confirm the stronger hydrogen bond between MA cations and the inorganic framework in $\mathrm{MAPbBr}_{3}$ than in $\mathrm{MAPbI}_{3}(0.06 \mathrm{eV}$ difference in MA binding energy in favor of $\mathrm{MAPbBr}_{3}$ ). Notably, FA experiences a higher rotational freedom compared to MA, as evidenced by ab initio molecular dynamics simulations, ${ }^{34}$ consistent with the reduced activation energy for cation reorientation observed here. Despite such trends, the relaxation times are shorter at all temperatures for $\mathrm{MAPbBr}_{3}$ in comparison with $\mathrm{MAPbI}_{3}$. This has been previously reported by Selig et al., ${ }^{35}$ and has been attributed to a higher probability of large angle jumps combined with tilting of the inorganic cage. ${ }^{12,35}$ In contrast to previous findings, ${ }^{35}$ for the mixed cation/halide, FAMA, we observe a much lower activation energy and relaxation times comparable to $\mathrm{MAPbI}_{3}$ at room temperature. Furthermore, the relaxation times for FAMA do not show an evident temperature dependence, while, as expected, for both $\mathrm{MAPbI}_{3}$ and $\mathrm{MAPbBr}_{3}, \tau$ is considerably slowed down upon cooling. We attribute the FAMA results to a combined effect of the rotational freedom in a larger crystal structure induced by the predominant presence of $\mathrm{FA}^{+}$cations and a reduced dipolar interaction between the MA ions.

Next, we can use the experimentally found relaxation times, to calculate the frequency-dependent values of $\varepsilon^{\prime \prime}$ using the Cole-Cole equation (see Figures S5 for $\varepsilon^{\prime}$ versus frequency). For $\mathrm{MAPbI}_{3}$ and $\mathrm{MAPbBr}_{3}$, these extrapolations are shown as solid lines for different temperatures in Figure 3, crossing our experimental values at $8.6 \mathrm{GHz}$. Interestingly, the maximum in dielectric loss shifts with temperature from frequencies much lower than $8.6 \mathrm{GHz}$ to values much higher than $8.6 \mathrm{GHz}$. This behavior explains our observed peaks for the dielectric loss at 220 and $200 \mathrm{~K}$ for $\mathrm{MAPbI}_{3}$ and $\mathrm{MAPbBr}_{3}$, respectively. The present interpretation agrees with the IS measurements; that is, $\varepsilon^{\prime \prime}$ continuously decreases with lower frequencies and drops below 2 at $4 \mathrm{kHz}$ for all investigated temperatures (see Figure S8). Furthermore, at these frequencies we observe a huge 

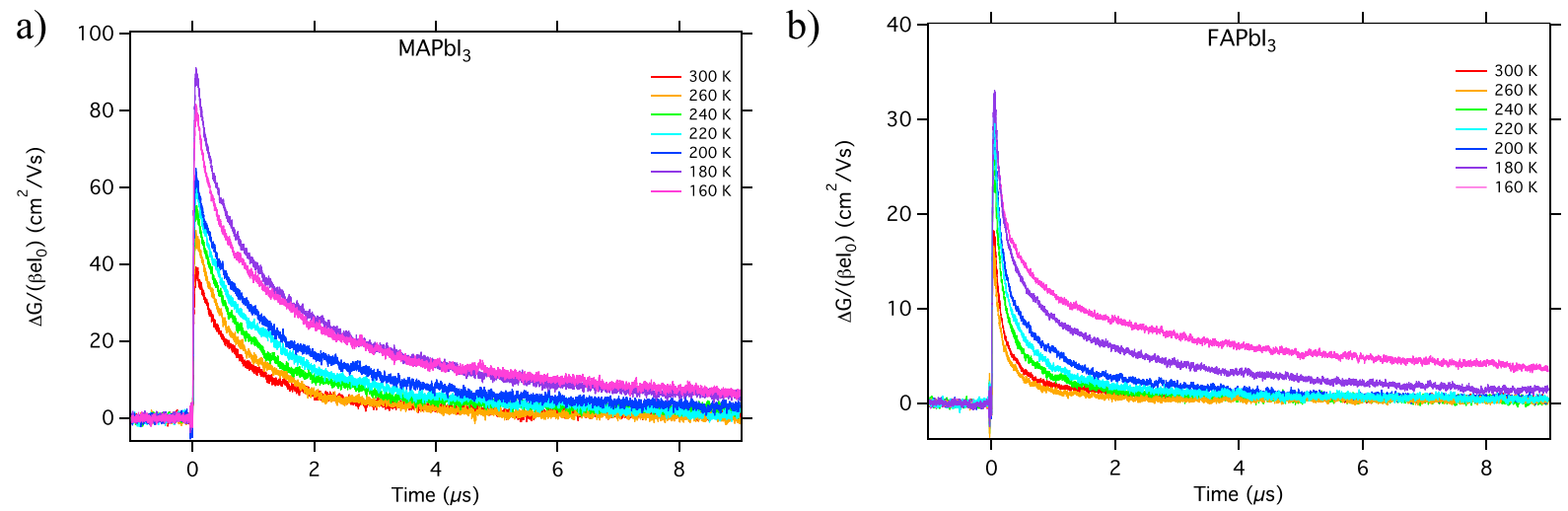

Figure 4. TRMC traces recorded on laser pulses with intensities of ca. $1-3 \times 10^{9}$ photons $/ \mathrm{cm}^{2}$ at $650 \mathrm{~nm}$ for $(\mathrm{a}) \mathrm{MAPbI}_{3}$ and $(\mathrm{b}) \mathrm{FAPbI}$.

difference in $\varepsilon^{\prime}$ between $\mathrm{MAPbI}_{3}$ and $\mathrm{FAPbI}_{3}$ (see Figure $\mathrm{S} 7$ ). Thus, we conclude that large, temperature-dependent variations in the real and imaginary parts of the dielectric constant are observed specifically in the $\mathrm{GHz}$ regime for MAbased MHPs.

As previously mentioned, the motion of the MA cation has been subject of research to understand the exceptional optoelectronic properties of $\mathrm{MAPbI}_{3}$. In particular, the possibility of enhanced charge carrier diffusion lengths as a result of weaker trapping ${ }^{7,11}$ and slower recombination due to defect and electronic screening ${ }^{11,36}$ are still debated. Over the years we have investigated the charge carrier decay kinetics of several MHPs, differing in composition and fabrication method. ${ }^{37-40}$ On the basis of the results obtained, some of us previously concluded that the organic cation does not play a major role in the charge carrier kinetics. ${ }^{38,41}$ In agreement with these findings, charge/lattice response due to formation of large polarons was found to have a similar subpicosecond time scale in both MA- and $\mathrm{Cs}-\mathrm{PbBr}_{3}$ perovskites. ${ }^{42}$

To verify if the rotational relaxation times affect the charge carrier dynamics, time-resolved microwave photoconductance (TRMC) traces are recorded at different temperatures and shown in Figure 4. Generally, all the MHP layers analyzed above $\left(\mathrm{CsPbI}_{3}\right.$ in Supporting Information) show similar trends on increasing the temperature from 160 to $300 \mathrm{~K}$ : (i) lower charge carrier mobilities and (ii) shorter charge carrier lifetimes. A detailed discussion of these findings can be found in previously published papers. ${ }^{43,44}$

With respect to the above discussion on the dielectric losses we can conclude the following: the temperature-dependent TRMC traces confirm that the changes in the dielectric losses observed with MC for the MA-based MHPs are not due to mobile charge carriers. If the changes in dielectric loss were due to mobile electrons and/or holes, the decay kinetics of MA-based samples should have been greatly affected, especially around 200-220 K. However, such behavior is not observed for $\mathrm{MAPbI}_{3}$, as shown in Figure 4. More importantly, the results reported here support the conclusion of a negligible impact of the nature of the A-site cation on the decay kinetics. In fact, despite the large change in dielectric loss, the temperature-dependent trend in mobility and lifetime of excess charge carriers in MA- and FA-based MHPs is very similar. On the other hand, no direct similarities can be found between $\mathrm{MAPbI}_{3}$ and $\mathrm{MAPbBr}_{3},{ }^{40,43}$ for which charge carrier mobility and recombination are substantially different (Figures $5 \mathrm{a}$ and S9a, respectively). Hence, from the present data no indications of reduced charge carrier recombination and/or defect screening by the MA are observed. A similar effect was previously discussed in the context of exciton screening, finding that the low-energy MA rotational dynamics barely affects the exciton binding energy in $\mathrm{MAPbI}_{3}$, which is instead significantly affected by phonons in the terahertz region. ${ }^{45}$

In this work, we have investigated the temperaturedependent microwave conductivity of several metal halide perovskite materials in the dark. Interestingly, all MA-based samples showed high dark conductivities in the $\mathrm{GHz}$ regime with distinctive temperature variations. In contrast, no appreciable dark conductivity can be observed at frequencies of $1 \mathrm{kHz}$ using IS measurements, which excludes ionic and electronic contributions. Therefore, we attribute the conductivities observed in the $\mathrm{GHz}$ regime to dielectric losses related to the rotation dynamics of the MA cation. Using the Cole-Cole model, we determined the activation energies and temperature-dependent relaxation times for different MAbased samples. On comparison of these results with the temperature-dependent photoconductivity, no correlation between dipolar rotational dynamics and light-induced charge carrier dynamics in MHPs was found. These results show that the huge changes in the dielectric constant at $\mathrm{GHz}$ frequencies do not impact the carrier dynamics due to the different time scales involved. Hence, we conclude that the fast carriers do not feel the slow-moving MA cations, despite the fact that the latter contribute to the overall value of the dielectric constant.

\section{ASSOCIATED CONTENT}

\section{S Supporting Information}

The Supporting Information is available free of charge on the ACS Publications website at DOI: 10.1021/acs.jpclett.9b02160.

Sample preparation, XRD and UV-vis, microwave conductance, dielectric model equation and parameters, impedance spectroscopy, and computational details (PDF)

\section{AUTHOR INFORMATION}

\section{Corresponding Authors}

*E-mail: E.Hutter@amolf.nl.

*E-mail: T.J.Savenije@tudelft.nl.

ORCID ${ }^{\circ}$

Daniele Meggiolaro: 0000-0001-9717-133X

Filippo De Angelis: 0000-0003-3833-1975

Samuel D. Stranks: 0000-0002-8303-7292 
Andreas Baumann: 0000-0002-9440-0456

Vladimir Dyakonov: 0000-0001-8725-9573

Eline M. Hutter: 0000-0002-5537-6545

Tom J. Savenije: 0000-0003-1435-9885

\section{Notes}

The authors declare no competing financial interest.

\section{ACKNOWLEDGMENTS}

V.M.C. and T.J.S. received funding from the Dutch Research Council (NWO) Grant Number 739.017.004. D.M., E.M., and F.D.A. acknowledge funding from the European Union's Horizon 2020 research and innovation programme under Grant Agreement No. 764047 of the ESPRESSO project. "Ministero dell'Istruzione dell'Università e della Ricerca (MIUR)" and "Università degli Studi di Perugia" are acknowledged for financial support through the program "Dipartimenti di Eccellenza 2018-2022" (Grant AMIS) to F.D.A. E.M.H. received funding from the Dutch Research Council (NWO) under the Echo Grant Number 712.014.007. S.D.S. acknowledges the Royal Society and Tata Group (UF150033). A.B. and M.F. acknowledge funding from the German Federal Ministry for Education and Research (BMBF) under Grant Number 03SF0514A/B of the HYPER project. V.D. acknowledges the Bavarian State Ministry of Science and Arts for funding of the Collaborative Research Network "Solar Technologies go Hybrid". A.B. works at the ZAE Bayern and is supported by the Bavarian Ministry of Economic Affairs, Energy and Technology.

\section{REFERENCES}

(1) Sun, J.; Wu, J.; Tong, X.; Lin, F.; Wang, Y.; Wang, Z. M. Organic/Inorganic Metal Halide Perovskite Optoelectronic Devices beyond Solar Cells. Adv. Sci. 2018, 5 (5), 1700780.

(2) De Wolf, S.; Holovsky, J.; Moon, S. J.; Löper, P.; Niesen, B.; Ledinsky, M.; Haug, F. J.; Yum, J. H.; Ballif, C. Organometallic Halide Perovskites: Sharp Optical Absorption Edge and Its Relation to Photovoltaic Performance. J. Phys. Chem. Lett. 2014, 5 (6), 10351039.

(3) Savenije, T. J.; Ponseca, C. S.; Kunneman, L.; Abdellah, M.; Zheng, K.; Tian, Y.; Zhu, Q.; Canton, S. E.; Scheblykin, I. G.; Pullerits, T.; et al. Thermally Activated Exciton Dissociation and Recombination Control the Carrier Dynamics in Organometal Halide Perovskite. J. Phys. Chem. Lett. 2014, 5 (13), 2189-2194.

(4) Umari, P.; Mosconi, E.; De Angelis, F. Infrared Dielectric Screening Determines the Low Exciton Binding Energy of MetalHalide Perovskites. J. Phys. Chem. Lett. 2018, 9 (3), 620-627.

(5) Bi, Y.; Hutter, E. M.; Fang, Y.; Dong, Q.; Huang, J.; Savenije, T. J. Charge Carrier Lifetimes Exceeding $15 \mathrm{Ms}$ in Methylammonium Lead Iodide Single Crystals. J. Phys. Chem. Lett. 2016, 7 (5), 923928.

(6) Hutter, E. M.; Eperon, G. E.; Stranks, S. D.; Savenije, T. J. Charge Carriers in Planar and Meso-Structured Organic-Inorganic Perovskites: Mobilities, Lifetimes, and Concentrations of Trap States. J. Phys. Chem. Lett. 2015, 6 (15), 3082-3090.

(7) Frost, J. M.; Butler, K. T.; Walsh, A. Molecular Ferroelectric Contributions to Anomalous Hysteresis in Hybrid Perovskite Solar Cells. APL Mater. 2014, 2 (8), 081506.

(8) Frost, J. M.; Butler, K. T.; Brivio, F.; Hendon, C. H.; van Schilfgaarde, M.; Walsh, A. Atomistic Origins of High-Performance in Hybrid Halide Perovskite Solar Cells. Nano Lett. 2014, 14 (5), 25842590.

(9) Herz, L. M. How Lattice Dynamics Moderate the Electronic Properties of Metal-Halide Perovskites. J. Phys. Chem. Lett. 2018, 9 (23), 6853-6863.
(10) Ma, J.; Wang, L. W. Nanoscale Charge Localization Induced by Random Orientations of Organic Molecules in Hybrid Perovskite $\mathrm{CH}_{3} \mathrm{NH}_{3} \mathrm{PbI}_{3}$. Nano Lett. 2015, 15 (1), 248-253.

(11) Anusca, I.; Balčiūnas, S.; Gemeiner, P.; Svirskas, Š.; Sanlialp, M.; Lackner, G.; Fettkenhauer, C.; Belovickis, J.; Samulionis, V.; Ivanov, M.; et al. Dielectric Response: Answer to Many Questions in the Methylammonium Lead Halide Solar Cell Absorbers. Adv. Energy Mater. 2017, 7 (19), 1700600.

(12) Gallop, N. P.; Selig, O.; Giubertoni, G.; Bakker, H. J.; Rezus, Y. L. A.; Frost, J. M.; Jansen, T. L. C.; Lovrincic, R.; Bakulin, A. A. Rotational Cation Dynamics in Metal Halide Perovskites: Effect on Phonons and Material Properties. J. Phys. Chem. Lett. 2018, 9 (20), 5987-5997.

(13) Mattoni, A.; Filippetti, A.; Saba, M. I.; Delugas, P. Methylammonium Rotational Dynamics in Lead Halide Perovskite by Classical Molecular Dynamics: The Role of Temperature. J. Phys. Chem. C 2015, 119 (30), 17421-17428.

(14) Kanno, S.; Imamura, Y.; Hada, M. Theoretical Study on Rotational Controllability of Organic Cations in Organic-Inorganic Hybrid Perovskites: Hydrogen Bonds and Halogen Substitution. J. Phys. Chem. C 2017, 121 (47), 26188-26195.

(15) Kanno, S.; Imamura, Y.; Saeki, A.; Hada, M. Rotational Energy Barriers and Relaxation Times of the Organic Cation in Cubic Methylammonium Lead/Tin Halide Perovskites from First Principles. J. Phys. Chem. C 2017, 121 (26), 14051-14059.

(16) Govinda, S.; Kore, B. P.; Bokdam, M.; Mahale, P.; Kumar, A.; Pal, S.; Bhattacharyya, B.; Lahnsteiner, J.; Kresse, G.; Franchini, C. Behavior of Methylammonium Dipoles in $\mathrm{MAPbX}_{3}(\mathrm{X}=\mathrm{Br}$ and I). $J$. Phys. Chem. Lett. 2017, 8, 4113.

(17) Chen, T.; Foley, B. J.; Ipek, B.; Tyagi, M.; Copley, J. R. D.; Brown, C. M.; Choi, J. J.; Lee, S. H. Rotational Dynamics of Organic Cations in the $\mathrm{CH}_{3} \mathrm{NH}_{3} \mathrm{PbI}_{3}$ Perovskite. Phys. Chem. Chem. Phys. 2015, 17 (46), 31278-31286.

(18) Li, J.; Bouchard, M.; Reiss, P.; Aldakov, D.; Pouget, S.; Demadrille, R.; Aumaitre, C.; Frick, B.; Djurado, D.; Rossi, M.; et al. Activation Energy of Organic Cation Rotation in $\mathrm{CH}_{3} \mathrm{NH}_{3} \mathrm{PbI}_{3}$ and $\mathrm{CD}_{3} \mathrm{NH}_{3} \mathrm{PbI}_{3}$ : Quasi-Elastic Neutron Scattering Measurements and First-Principles Analysis Including Nuclear Quantum Effects. J. Phys. Chem. Lett. 2018, 9 (14), 3969-3977.

(19) Onoda-Yamamuro, N.; Matsuo, T.; Suga, H. Calorimetric and IR Spectroscopic Studies of Phase Transitions in Methylammonium Trihalogenoplumbates (II). J. Phys. Chem. Solids 1990, 51 (12), $1383-1395$.

(20) Quarti, C.; Grancini, G.; Mosconi, E.; Bruno, P.; Ball, J. M.; Lee, M. M.; Snaith, H. J.; Petrozza, A.; De Angelis, F. The Raman Spectrum of the $\mathrm{CH}_{3} \mathrm{NH}_{3} \mathrm{PbI}_{3}$ Hybrid Perovskite: Interplay of Theory and Experiment. J. Phys. Chem. Lett. 2014, 5 (2), 279-284.

(21) Hoque, M. N. F.; Yang, M.; Li, Z.; Islam, N.; Pan, X.; Zhu, K.; Fan, Z. Polarization and Dielectric Study of Methylammonium Lead Iodide Thin Film to Reveal Its Nonferroelectric Nature under Solar Cell Operating Conditions. ACS Energy Lett. 2016, 1 (1), 142-149.

(22) Poglitsch, A.; Weber, D. Dynamic Disorder in Methylammoniumtrihalogenoplumbates (II) Observed by Millimeter-Wave Spectroscopy. J. Chem. Phys. 1987, 87 (11), 6373-6378.

(23) Yaffe, O.; Guo, Y.; Tan, L. Z.; Egger, D. A.; Hull, T.; Stoumpos, C. C.; Zheng, F.; Heinz, T. F.; Kronik, L.; Kanatzidis, M. G.; et al. Local Polar Fluctuations in Lead Halide Perovskite Crystals. Phys. Rev. Lett. 2017, 118 (13), 1-6.

(24) Stroppa, A.; Quarti, C.; De Angelis, F.; Picozzi, S. Ferroelectric Polarization of $\mathrm{CH}_{3} \mathrm{NH}_{3} \mathrm{PbI}_{3}$ : A Detailed Study Based on Density Functional Theory and Symmetry Mode Analysis. J. Phys. Chem. Lett. 2015, 6 (12), 2223-2231.

(25) Quarti, C.; Mosconi, E.; De Angelis, F. Interplay of Orientational Order and Electronic Structure in Methylammonium Lead Iodide: Implications for Solar Cell Operation. Chem. Mater. 2014, 26 (22), 6557-6569.

(26) Piet, J. J.; Schuddeboom, W.; Wegewijs, B. R.; Grozema, F. C.; Warman, J. M. Symmetry Breaking in the Relaxed $S_{1}$ Excited State of 
Bianthryl Derivatives in Weakly Polar Solvents. J. Am. Chem. Soc. 2001, 123 (22), 5337-5347.

(27) Fravventura, M. C.; Deligiannis, D.; Schins, J. M.; Siebbeles, L. D. A.; Savenije, T. J. What Limits Photoconductance in Anatase $\mathrm{TiO}_{2}$ Nanostructures? A Real and Imaginary Microwave Conductance Study. J. Phys. Chem. C 2013, 117 (16), 8032-8040.

(28) Cole, K. S.; Cole, R. H. Dispersion and Absorption in Dielectrics. J. Chem. Phys. 1941, 9 (1913), 341-351.

(29) Schins, J. M.; Talgorn, E. Conductive Response of a PhotoExcited Sample in a Radio-Frequent Driven Resonance Cavity. Rev. Sci. Instrum. 2011, 82 (6), 064703.

(30) Azpiroz, J. M.; Mosconi, E.; Bisquert, J.; De Angelis, F. Defect Migration in Methylammonium Lead Iodide and Its Role in Perovskite Solar Cell Operation. Energy Environ. Sci. 2015, 8 (7), 2118-2127.

(31) Frost, J. M.; Walsh, A. What Is Moving in Hybrid Halide Perovskite Solar Cells? Acc. Chem. Res. 2016, 49 (3), 528-535.

(32) Küçükçelebi, H.; Durmuş, H.; Deryal, A.; Taşer, M.; Karakaya, N. Activation Energy of Polarization Due to Electrical Conductivity and Dipole Rotation in Purified Ca-Bentonite. Appl. Clay Sci. 2012, 62-63 (March 2019), 70-79.

(33) Davidson, D. W.; Cole, R. H. Dielectric Relaxation in Glycerol, Propylene Glycol, and n-Propanol. J. Chem. Phys. 1951, 19 (12), 1484.

(34) Quarti, C.; Mosconi, E.; De Angelis, F. Structural and Electronic Properties of Organo-Halide Hybrid Perovskites from Ab Initio Molecular Dynamics. Phys. Chem. Chem. Phys. 2015, 17 (14), 9394-9409.

(35) Selig, O.; Sadhanala, A.; Müller, C.; Lovrincic, R.; Chen, Z.; Rezus, Y. L. A.; Frost, J. M.; Jansen, T. L. C.; Bakulin, A. A. Organic Cation Rotation and Immobilization in Pure and Mixed Methylammonium Lead-Halide Perovskites. J. Am. Chem. Soc. 2017, 139 (11), 4068-4074.

(36) Milot, R. L.; Eperon, G. E.; Snaith, H. J.; Johnston, M. B.; Herz, L. M. Temperature-Dependent Charge-Carrier Dynamics in $\mathrm{CH}_{3} \mathrm{NH}_{3} \mathrm{PbI}_{3}$ Perovskite Thin Films. Adv. Funct. Mater. 2015, 25 (39), 6218-6227.

(37) Hutter, E. M.; Sutton, R. J.; Chandrashekar, S.; Abdi-Jalebi, M.; Stranks, S. D.; Snaith, H. J.; Savenije, T. J. Vapour-Deposited Cesium Lead Iodide Perovskites: Microsecond Charge Carrier Lifetimes and Enhanced Photovoltaic Performance. ACS Energy Lett. 2017, 2, 1901-1908.

(38) Hu, Y.; Hutter, E. M.; Rieder, P.; Grill, I.; Hanisch, J.; Aygüler, M. F.; Hufnagel, A. G.; Handloser, M.; Bein, T.; Hartschuh, A.; et al. Understanding the Role of Cesium and Rubidium Additives in Perovskite Solar Cells: Trap States, Charge Transport, and Recombination. Adv. Energy Mater. 2018, 8, 1703057.

(39) Abdi-Jalebi, M.; Andaji-Garmaroudi, Z.; Cacovich, S.; Stavrakas, C.; Philippe, B.; Richter, J. M.; Alsari, M.; Booker, E. P.; Hutter, E. M.; Pearson, A. J.; et al. Maximizing and Stabilizing Luminescence from Halide Perovskites with Potassium Passivation. Nature 2018, 555, 497.

(40) Guo, D.; Bartesaghi, D.; Wei, H.; Hutter, E. M.; Huang, J.; Savenije, T. J. Photoluminescence from Radiative Surface States and Excitons in Methylammonium Lead Bromide Perovskites. J. Phys. Chem. Lett. 2017, 8 (17), 4258-4263.

(41) Hutter, E. M.; Savenije, T. J. Thermally Activated SecondOrder Recombination Hints toward Indirect Recombination in Fully Inorganic CsPbl(3) Perovskites. ACS Energy Lett. 2018, 3, 2068.

(42) Trinh, M. T.; Miyata, K.; Joshi, P. P.; Jones, S. C.; De Angelis, F.; Mosconi, E.; Zhu, X.-Y.; Meggiolaro, D. Large Polarons in Lead Halide Perovskites. Sci. Adv. 2017, 3 (8), e1701217.

(43) Hutter, E. M.; Gélvez-Rueda, M. C.; Osherov, A.; Bulović, V.; Grozema, F. C.; Stranks, S. D.; Savenije, T. J. Direct-Indirect Character of the Bandgap in Methylammonium Lead Iodide Perovskite. Nat. Mater. 2017, 16 (1), 115-120.

(44) Osherov, A.; Hutter, E. M.; Galkowski, K.; Brenes, R.; Maude, D. K.; Nicholas, R. J.; Plochocka, P.; Bulović, V.; Savenije, T. J.; Stranks, S. D. The Impact of Phase Retention on the Structural and
Optoelectronic Properties of Metal Halide Perovskites. Adv. Mater. 2016, 28 (48), 10757-10763.

(45) Umari, P.; Mosconi, E.; De Angelis, F. Infrared Dielectric Screening Determines the Low Exciton Binding Energy of MetalHalide Perovskites. J. Phys. Chem. Lett. 2018, 9 (3), 620-627. 